\title{
A Lyman-alpha-based (VUV) plasma density fluctuation diagnostic design
}

\author{
G. R. McKee, ${ }^{\text {a) }}$ R. J. Fonck, C. Fenzi, and B. P. Leslie \\ University of Wisconsin-Madison, 1500 Engineering Drive, Madison, Wisconsin 53706
}

(Presented on 21 June 2000)

\begin{abstract}
The benchmark design for a high spatial resolution, high sensitivity, long-wavelength density fluctuation diagnostic that observes collisionally induced Lyman-alpha emissions $\left(\lambda_{0} \approx 121.6 \mathrm{~nm}\right)$ from neutral beam atoms is presented. The sensitivity to plasma fluctuations at $\mathrm{Ly}_{\alpha}$ is estimated to be about 50 times higher than at $\mathrm{D}_{\alpha}$. This dramatically increases the photon-noise-limited signal-to-noise ratio. Design criteria include a spectral resolution of $\Delta \lambda \approx 0.5 \mathrm{~nm}$ at $\mathrm{Ly}_{\alpha}$, to isolate the Doppler-shifted beam emission from the bright edge $\mathrm{L}_{\alpha}$ emission; high quantum efficiency detectors; high optical throughput; multichannel capability in the radial and poloidal directions; spatial resolution near $1 \mathrm{~cm}$ in the radial and poloidal directions to measure fluctuations in the wave number range $0.1 \leqslant k_{r}, k_{\theta} \leqslant 3 \mathrm{~cm}^{-1}$. A system design based on a Czerny-Turner monochromator is considered. (C) 2001 American Institute of Physics. [DOI: 10.1063/1.1323246]
\end{abstract}

\section{INTRODUCTION}

To investigate and more thoroughly understand turbulent processes in plasmas, a broad array of fluctuation diagnostics have been deployed on various tokamak and other plasma confinement experiments. ${ }^{1}$ These diagnostic measurements have helped to characterize and understand turbulence ${ }^{2,3}$ which in turn has enabled turbulent transport reduction in various plasma operational modes. ${ }^{4,5}$ Despite increased understanding of turbulence, many critical issues remain to be investigated, and the study of plasma turbulence continues to be a fascinating and complex field of scientific inquiry. Specific topics requiring further research include understanding the LH transition, nonlinear energy cascade processes, $\mathrm{E} \times \mathrm{B}$ shear suppression of turbulence, as well as particle and electron transport processes.

The beam emission spectroscopy (BES) density fluctuation diagnostic has provided useful turbulence measurements and has been implemented on several tokamaks (TFTR, ${ }^{6}$ DIII-D, $\left.{ }^{7} \mathrm{C}-\mathrm{MOD}^{8}\right)$. The existing BES system measures localized long-wavelength $\left(k_{\perp}<3 \mathrm{~cm}^{-1}\right)$ density fluctuations by observing the Doppler-shifted $n=3-2 \mathrm{D}_{\alpha}$ transition ( $\lambda$ $\approx 656 \mathrm{~nm}$ ) arising from collisional excitation of the heating neutral beams. ${ }^{9}$ BES measurements have provided measurements of the $S\left(k_{r}\right)$ and $S\left(k_{\theta}\right)$ spectra, ${ }^{3}$ fluctuation amplitudes and profiles, radial and poloidal correlation lengths, decorrelation times, two-dimensional structure of turbulent eddies ${ }^{10}$ and energy cascades. ${ }^{11,12}$

One limitation of the BES system has been the photonnoise-limited signal-to-noise ratio for such measurements, particularly in higher confinement regimes in advanced tokamak plasmas ${ }^{13}$ and in the deep plasma core where beam attenuation and low inherent fluctuation levels limit sensitivity. Figure 1 shows a typical density fluctuation profile in an L-mode DIII-D plasma indicating the rapid decrease in fluctuations from the edge of the plasma, where fluctuation am-

${ }^{a)}$ Current address: General Atomics, 13-367, P.O. Box 85608, San Diego, CA 92186-5608. plitudes $(\widetilde{n} / n)$ are typically of order $1 \%-10 \%$, into $\rho=0.7$, where fluctuations are of order $\leqslant 0.1 \%$. Thus a wide dynamic range and high sensitivity are required.

One approach to dramatically increasing the signal-tonoise ratio of density fluctuation measurements while retaining the high spatial resolution of BES is to measure the Doppler shifted $n=2-1 \mathrm{Ly}_{\alpha}$ transition $(\lambda \approx 121 \mathrm{~nm})$ of the neutral beam atoms. The critical advantage to this approach is that the photon flux of the $\mathrm{Ly}_{\alpha}$ transition is between one and two orders of magnitude higher than for $\mathrm{D}_{\alpha}$. This dramatic increase in brightness has the potential to substantially improve signal quality of density fluctuation measurements.

Along with the opportunities for significant signal enhancement come technical challenges of designing a diagnostic system for the vacuum ultraviolet (VUV) spectral region where optics, spectrometers, and detectors are generally not as efficient or highly optimized as in the visible spectrum. This paper discusses aspects of the conceptual design of a viable $\mathrm{Ly}_{\alpha}$-based VUV BES density fluctuation diagnostic system.

The motivation for a VUV BES system is the higher photon flux from the $n=2-1$ transition relative to the $n$ $=3-2$ transition. The photon radiance is estimated as

$$
I_{m n}=\frac{1}{4 \pi} L A_{m n} n_{b m}^{*}
$$

with $L$ the path length of the optical sightline through the emission zone, $A_{m n}$ the $m \rightarrow n$ atomic transition rate, $n_{b m}^{*}$ the density of beam atoms in the $m$ th excited state. $n_{b m}^{*}$ is calculated using a multistage beam excitation code. ${ }^{14}$ Table I shows the comparison of the critical parameters for the Balmer $\mathrm{D}_{\alpha}$ transition and the Lyman-alpha transition of beam atoms in a typical plasma $\left(n_{e}=4 \times 10^{19} \mathrm{~m}^{-3}, T_{e}=T_{i}\right.$ $=5 \mathrm{keV}$ ). The resulting difference in photon emission rates is the product of these numbers, showing about a factor of 25 larger photon flux for $\mathrm{Ly}_{\alpha}$. Furthermore, the sensitivity to density fluctuations from the beam emission intensity is higher at $\mathrm{Ly}_{\alpha}$. Intensity $(d I)$ and density $(d n)$ fluctuations are 


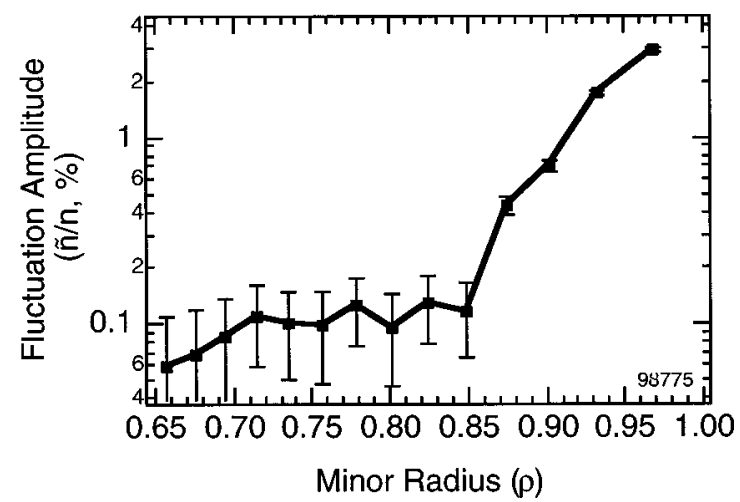

FIG. 1. Typical radial profile of fluctuation amplitudes with logarithmic $y$ scale, showing wide dynamic range required.

related as $d n / n=C^{*} d I / I$, with $C$ typically near 3 for $\mathrm{D}_{\alpha}$ and about 1.5 for $\mathrm{Ly}_{\alpha}$, making the measurement more sensitive to plasma fluctuations by a factor of 2 , thus increasing the total fluctuation sensitivity by an estimated factor of $50 .^{9}$

A secondary advantage is somewhat improved spatial resolution. At typical neutral beam energies (40 keV/amu), beam particles travel a finite distance during the atomic transition time. Because of the significantly larger atomic transition rate for the $\mathrm{Ly}_{\alpha}$ transition, this distance is about $0.4 \mathrm{~cm}$ relative to $1.5-2 \mathrm{~cm}$. for the $\mathrm{D}_{\alpha}$ transition. ${ }^{9}$ The imaged region is likely to be around $1 \mathrm{~cm}$ in radial and poloidal extent so the spatial resolution is determined almost entirely by the image spot size.

In order to measure turbulent density fluctuations with $\mathrm{Ly}_{\alpha}$ emissions, the diagnostic system must satisfy several criteria. The emission must be spectrally isolated with the most stringent requirement being rejection of the edge $\mathrm{Ly}_{\alpha}$ emission $\left(\lambda_{0}=121.6 \mathrm{~nm}\right)$ arising from collisional excitation of recycling neutral deuterium atoms, while accepting nearly all of the beam emission. The beam velocity (2.7 $\times 10^{6} \mathrm{~m} / \mathrm{s}$ ) blueshifts this emission by between 0.3 and 0.6 $\mathrm{nm}$, depending on the neutral beam-sightline geometry at the radial position being observed. A spectral resolution of $\Delta \lambda$ $=0.5 \mathrm{~nm}$ is sufficient for most applications. The spatial resolution of one channel will be $\Delta r \leqslant 1.0 \mathrm{~cm}$, and $\Delta z \leqslant 2.0 \mathrm{~cm}$. These numbers are specified by the typical radial and poloidal correlation lengths of plasma turbulence, with $L_{c, r}$ $\geqslant 1.5 \mathrm{~cm}$, and $L_{c, \theta} \geqslant 3.0 \mathrm{~cm}$ under most plasma conditions encountered on DIII-D. The beam-sightline volume is extended in the toroidal direction over tens of $\mathrm{cm}$. This is acceptable since toroidal correlation lengths have been shown to be of the order of the plasma major radius, and thus long compared to these dimensions. The temporal resolution is dictated by turbulent flow velocities and eddy structure di-

TABLE I. Relative photon emission rates and fluctuation sensitivity for $\mathrm{D}_{\alpha}$ and $\mathrm{LY}_{\alpha}$.

\begin{tabular}{lccc}
\hline \hline \multicolumn{1}{c}{ Transition } & Lyman-alpha & Balmer-alpha & Ratio \\
\hline Wavelength $(\mathrm{nm})$ & $121(\mathrm{VUV})$ & $656($ visible $)$ & \\
Transition rate $\left(\mathrm{s}^{-1}\right)$ & $4.6 \times 10^{8}$ & $4.4 \times 10^{7}$ & 11 \\
State population density & 0.0037 & 0.0015 & 2.5 \\
Fluctuation sensitivity & 0.45 & 0.3 & 2 \\
\hline \hline
\end{tabular}

mensions as seen in the laboratory frame. Turbulent eddies have been shown experimentally to propagate near the local $E \times B$ velocity, typically several $\mathrm{km} / \mathrm{s}$ or faster. Structures of order of the correlation lengths discussed above would then be seen in the laboratory frame as fluctuations on the order of a few hundred $\mathrm{kHz}$. Thus a detector sampling rate of at least $1 \mathrm{MHz}$ is required to adequately measure turbulence.

\section{CONCEPTUAL DESIGN}

The design of a spectroscopic system to view the $\mathrm{Ly}_{\alpha}$ transition of the neutral beam atoms is entirely different from that which views the visible $\mathrm{D}_{\alpha}$ transition. ${ }^{7}$ The visible system relies on low $f /$ number lenses, optical fibers, interference filters, and photodiode detectors. Since $\mathrm{Ly}_{\alpha}$ photons do not pass through air or optical fibers, the spectroscopic system design is entirely in vacuum and situated in close physical proximity to the tokamak. The design consists of entirely reflective optics. Imaging optics would focus the beam emission region onto the entrance slit of a fast monochromator optimized for $\mathrm{Ly}_{\alpha}$. A VUV photodiode detector array is located at the output spectral image plane of the spectrometer. Signal conditioning electronics ${ }^{15}$ would amplify and filter the signal to be digitized. This design study is undertaken to determine the feasibility and scope of such a VUV-BES diagnostic. The individual components of this conceptual design are discussed in the following sections.

\section{A. Input imaging optics}

An input reflective optics array will be used to relay an image of the beam-plasma volume to the entrance slit of the spectrometer. There are no refractive materials with sufficient transmission at $\mathrm{Ly}_{\alpha}$ to allow for lens imaging. $\mathrm{LiF}$ and $\mathrm{MgF}_{2}$ windows of order 1-2 mm could be used, but the thickness is not sufficient for a lens, thus reflective (achromatic) optics are to be used. Two optical surfaces would image the observed spatial region to the entrance slit of a spectrometer, and demagnify the image by a factor of roughly 4 . Two optics also provide the flexibility to reduce spherical and comatic aberrations as well as astigmatism. Each optic would consist of a glass substrate coated with an aluminum layer. The aluminum is in turn coated with a layer of $\mathrm{MgF}_{2}$ which serves to prevent oxidation of the aluminum, and is applied with a thickness (about $250 \AA$ ) that optimizes reflectivity at $\mathrm{Ly}_{\alpha}$ through constructive interference of reflections at the two surfaces. The estimated reflectivity is near $78 \%$.

A design for this input optical assembly is shown in Fig. 2. The relatively high speed of this array leads to significant spherical, coma and astigmatic aberrations. A ray tracing program (BEAM4) is used to design these optics and minimize aberrations. The imaged region in the plasma for one spatial channel will be $0.8 \mathrm{~cm}$ (radially) by $1.2 \mathrm{~cm}$ (poloidally), and the image at the slit entrance will then be 2 $\times 3 \mathrm{~mm}$. The point-to-point blur at the image must therefore be kept to fractions of a mm. Simple spherical optics do not achieve this, with aberrations leading to a rms blur radius for point-to-point imaging of near $2.5 \mathrm{~mm}$. The optical design for both optics here is a toroidal mirror. The first with a 


\section{Toric collimating mirror}

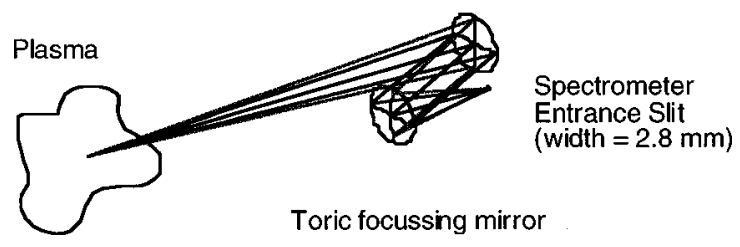

FIG. 2. Input optics array images the plasma volume to the spectrometer entrance slit with asymmetric demagnification (in plane defined by optical axis, and perpendicular) using toric mirrors.

radius of curvature of $2.04 \mathrm{~m}$ in the plane defined by the optical axis, and a $1.26 \mathrm{~m}$ radius of curvature in the perpendicular plane. The second optic has a radius of curvature of $6.4 \mathrm{~m}$ in the optical axis plane, and $0.95 \mathrm{~m}$ in the perpendicular plane. These toric shapes significantly reduce aberrations. The rms blur radius with these toroidal surfaces is near $0.2 \mathrm{~mm}$, thus achieving the necessary image quality. In addition, these mirrors allow for asymmetric demagnification so that a square region can be imaged to a rectangular shape that couples more effectively to the spectrometer if so desired.

It is also noted that these optics have high reflectivity at $\mathrm{D}_{\alpha}$ in the visible region. Since the reflective optics are achromatic, the imaging characteristics are the same for $\mathrm{D}_{\alpha}$ and $\mathrm{Ly}_{\alpha}$. This allows the possibility of maintaining the existing visible BES system along with a high sensitivity VUV system at the same port structure on the DIII-D tokamak.

\section{B. Monochromator}

Various spectrometer/monochromator designs have been developed and optimized for use in the VUV spectral region, including such configurations as the Seya-Namioka, Czerny-Turner, Monk-Giliesson, and Wadsworth designs. ${ }^{16}$ To get an overall scope of the spectrometer design requirements, we consider a Czerny-Turner system, although this would not necessarily be the final design implementation. To realize the benefits of the $\mathrm{Ly}_{\alpha}$ photon flux, it is necessary to implement a fast spectrometer. We thus examine an $f / 2$ configuration, exceptionally fast for a Czerny-Turner, and consider the spectral and spatial resolution capabilities. The imaged plasma area should have a dimension of $8 \mathrm{~mm}$ radially by $12 \mathrm{~mm}$ poloidally, with the input optics demagnifying the image by a factor of near 4 , thus the slit width, corresponding to the poloidal direction, is $3 \mathrm{~mm}$ and the slit height 2 $\mathrm{mm}$ for one spatial channel. Additional spatial (radial) channel images would be arrayed up and down the slit, separated by $2 \mathrm{~mm}$ each. These specific dimensions are chosen to match available detector arrays, discussed in the following section.

In order to achieve the necessary spectral resolution with the wide entrance slits, the spectrometer would have a focal length of $0.5 \mathrm{~m}$, and the grating would have $5760 \mathrm{~g} / \mathrm{mm}$ and be operated in second order. This high line-density grating, which would have dimensions of $0.25 \times 0.3 \mathrm{~m}$, can be manufactured using holographic techniques (Jobin-Yvon has manufactured similar gratings for astrophysical applica-
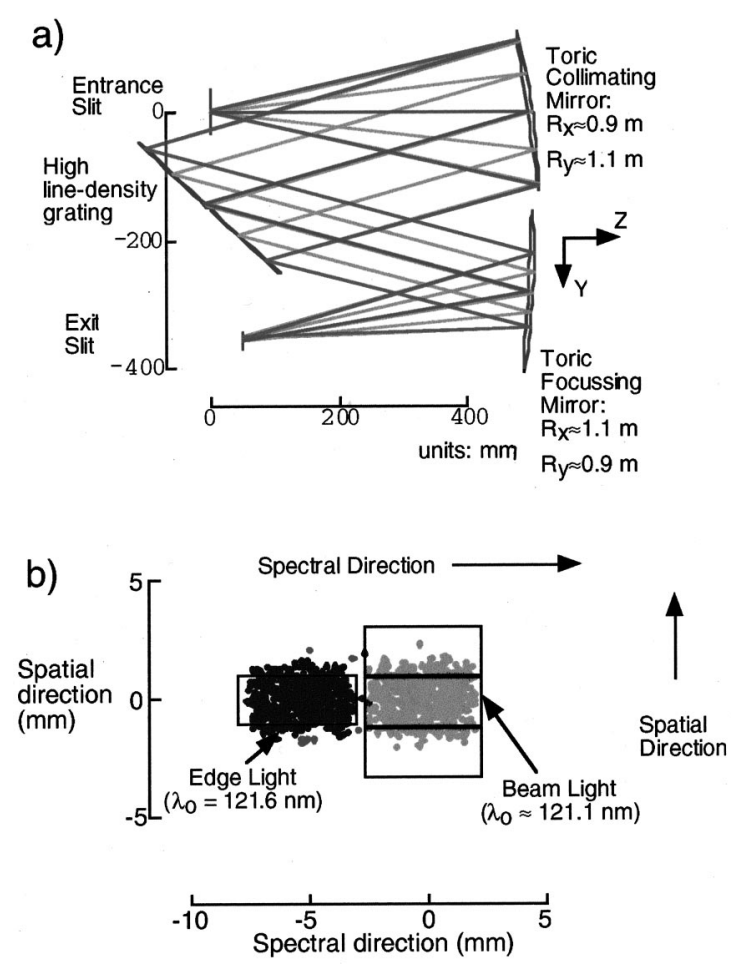

FIG. 3. (a) A fast Czerny-Turner layout with principle components. Parameters are $f=0.5 \mathrm{~m}, f / 2,5760 \mathrm{~g} / \mathrm{mm}$ grating (second order), $0.08 \mathrm{nn} / \mathrm{mm}$ dispersion, resolution of $0.5 \mathrm{~nm}$., toric mirrors; (b) ray tracing scatter plots showing effective spectral separation of edge and beam emission in the spectral (horizontal) direction with $2.8 \mathrm{~mm}$ entrance slits, and adequate spatial channel separation in the spatial (vertical) direction, with each channel indicated by stacked rectangular boxes.

tions). It will be challenging to optimize the groove profile to achieve maximum diffraction into second order. Ion etching techniques may allow for groove shaping. Second order operation is required to achieve the necessary dispersion of $0.08 \mathrm{~nm} / \mathrm{mm}$. The efficiency of the grating is estimated to be $25 \%$ for unpolarized input light, impacting system throughput. The magnification of the input slit width on the spectral direction of the focal plane is near 1.7, resulting in an output image of $5 \mathrm{~mm}$ (spectral) by $2 \mathrm{~mm}$ (spatial). As will be seen next, this matches well with commercially available detector arrays. A schematic of the spectrometer system is shown in Fig. 3(a). Another option is to utilize a $3600 \mathrm{~g} / \mathrm{mm}$ grating of the same dimensions in third order. Square groove profiles disperse significant power fraction into third order. The spectral resolution, dispersion and magnification would be similar.

The $f / 2$, high speed design of the spectrometer leads to significant aberrations. Spherical optics, as with the input optical array, would yield unacceptable image quality. The necessary spectral resolution of $0.5 \mathrm{~nm}$, and spatial resolution of $2 \mathrm{~mm}$ along the entrance slit, are achieved using toroidal collimating and focusing mirrors in the CzernyTurner design. The $0.5 \mathrm{~m}$ focal length leads to mirror radii of curvature of nominally $1.0 \mathrm{~m}$. An optimized toric design for the first (collimating) mirror has a roughly 1.1 radius of curvature $\left(R_{c}\right)$ in the plane of the optical axis and and $R_{c}$ $=0.9 \mathrm{~m}$ the perpendicular plane. The second (focusing) mirror has radii of curvature near 0.9 and $1.1 \mathrm{~m}$, respectively. 
The imaging and spectral resolution then allow for complete isolation of the edge $\mathrm{Ly}_{\alpha}$ and full-energy beam $\mathrm{Ly}_{\alpha}(0.5 \mathrm{~nm}$ to the blue) component, with partial observation of the half and third energy components. Ray tracing scatter plot diagrams of the output spectral plane are shown in Fig. 3(b).

\section{Detector}

Detector quantum efficiencies near $\mathrm{Ly}_{\alpha}$ have historically been quite low $(\ll 1)$ due to strong absorption of $\mathrm{Ly}_{\alpha}$ photons in most window materials. Demand for more efficient detectors by the astrophysical and high energy physics community has spurred the development of more advanced detectors. A particular photodiode (AXUV Series, International Radiation Detectors, Inc.) has been identified that has near unity quantum efficiency at $\mathrm{Ly}_{\alpha}$. This high efficiency is due to two unique design features: no dead region in the bulk semiconductor that would allow for recombination of photoelectrons, and extremely thin windows $(\sim 5 \mathrm{~nm})$. These silicon detectors have a thin silicon dioxide window that is normally strongly absorbing to $\mathrm{Ly}_{\alpha}$ photons. Nitrided silicon dioxide further improves $\mathrm{Ly}_{\alpha}$ sensitivity. It is believed that the charge resulting from photons absorbed in the window can largely still be observed by the detection circuit, allowing near theoretical quantum efficiencies, although the detector physics is not fully understood at this wavelength.

This photodiode detector is commercially available in an array format with individual pixels of dimensions 2 $\times 5 \mathrm{~mm}$, matching precisely to the output dimensions of the image for one spatial channel. 16 pixels are available in the detector, allowing for 16 spatial channels in one dimension (radial or poloidal) to be observed simultaneously. The photodiode response time is $\leqslant 0.5 \mu$ s with a capacitance of $\leqslant 0.2$ $\mathrm{nF}$, thus satisfying the temporal response requirements. It is crucial to minimize the detector capacitance as noise in the preamplifier circuitry is proportional to capacitance. ${ }^{15}$ Biasing the detector at up to $8 \mathrm{~V}$ further reduces capacitance and improves temporal response, while slightly increasing dark current. The dark current should be insignificant compared to the photocurrent and thus not a concern.

\section{SUMMARY}

A conceptual design for a VUV-BES density fluctuation diagnostic system to explore plasma turbulence in magnetically confined fusion plasmas has been presented. The pro- posed diagnostic observes the Doppler-shifted Lyman alpha emissions near $121 \mathrm{~nm}$ of collisionally excited heating neutral beam atoms. The $\mathrm{Ly}_{\alpha}$ transition is roughly 50 times more sensitive than the $\mathrm{D}_{\alpha}$ transition to density fluctuations and achieves high spatial resolution. Given differences in optical transmission, dispersion efficiency and quantum efficiency, the existing visible BES system is estimated to have roughly three times greater throughput than this VUV-BES design. Coupled with the higher photon flux and fluctuation sensitivity, the VUV system should then have more than an order of magnitude increase in sensitivity compared to the visible system. Further design considerations will investigate multilayer coated optics to image and spectrally filter plasma emissions, alternative spectrometer configurations, as well as multiple resonance reflectors to isolate the edge and beam emission, and seek to minimize optical aberrations to achieve the highest spatial and spectral resolution possible.

\footnotetext{
${ }^{1}$ N. Bretz, Rev. Sci. Instrum. 68, 2927 (1997).

${ }^{2}$ B. A. Carreras, IEEE Trans. Plasma Sci. 25, 1281 (1997).

${ }^{3}$ R. J. Fonck, G. Cosby, R. D. Durst, S. F. Paul, N. Bretz, S. Scott, E. Synakowski, and G. Taylor, Phys. Rev. Lett. 70, 3736 (1993).

${ }^{4}$ G. R. McKee, M. Murakami, J. A. Boedo, N. H. Brooks, K. H. Burrell, D. R. Ernst, R. J. Fonck, G. L. Jackson, M. Jakubowski, R. J. LaHaye et al., Phys. Plasmas 7, 1870 (2000).

${ }^{5}$ C. M. Greenfield, J. C. DeBoo, T. C. Luce, B. W. Stallard, E. J. Synakowski, L. R. Baylor, K. H. Burrell, T. A. Casper, E. J. Doyle, D. R. Ernst et al., Phys. Plasmas 7, 1959 (2000).

${ }^{6}$ R. D. Durst, R. J. Fonck, G. Cosby, H. Evensen, and S. F. Paul, Rev. Sci. Instrum. 63, 4907 (1992).

${ }^{7}$ G. McKee, R. Ashley, R. Durst, R. Fonck, M. Jakubowski, K. Tritz, K. Burrell, C. Greenfield, and J. Robinson, Rev. Sci. Instrum. 70, 913 (1999).

${ }^{8}$ R. V. Bravenec, M. B. Sampsell, W. L. Rowan, E. C. Eisner, D. M. Patterson, N. L. Bretz, A. E. Hubbard, J. H. Irby, E. S. Marmar, and J. L. Terry, Bull. Am. Phys. Soc. 44, 207 (1999).

${ }^{9}$ R. J. Fonck, P. A. Dupperex, and S. F. Paul, Rev. Sci. Instrum. 61, 3487 (1990).

${ }^{10}$ C. Fenzi, R. Fonck, and G. McKee, Rev. Sci. Instrum. (these proceedings).

${ }^{11}$ J. S. Kim, R. D. Durst, R. J. Fonck, E. Fernandez, A. Ware, and P. W. Terry, Phys. Plasmas 3, 3998 (1996).

${ }^{12}$ J. S. Kim, R. J. Fonck, R. D. Durst, E. Fernandez, P. W. Terry, S. F. Paul, and M. C. Zarnstorff, Phys. Rev. Lett. 79, 841 (1997).

${ }^{13}$ K. H. Burrell, Rev. Sci. Instrum. (these proceedings).

${ }^{14}$ T. A. Gianakon, R. J. Fonck, J. D. Callen, R. D. Durst, and J. S. Kim, Rev. Sci. Instrum. 63, 4931 (1992)

${ }^{15}$ R. J. Fonck, R. Ashley, R. Durst, S. F. Paul, and G. Renda, Rev. Sci. Instrum. 63, 4924 (1992).

${ }^{16}$ J. A. Samson, Vacuum Ultraviolet Spectroscopy I \& II, Experimental Methods in the Physical Sciences (Academic, San Diego, 1998), Vols. 31
} and 32. 